BMJ Open

Sport \&

Exercise

Medicine

\title{
Association between fasting insulin and high-sensitivity $C$ reactive protein in Korean adults
}

\author{
June S Yang, ${ }^{1}$ Jeffry N Gerber, ${ }^{2}$ Hyun J You ${ }^{3}$
}

To cite: Yang JS, Gerber JN, You HJ. Association between fasting insulin and highsensitivity $C$ reactive protein in Korean adults. BMJ Open Sport Exerc Med 2017;3 e000236. doi:10.1136/ bmjsem-2017-000236

Accepted 9 April 2017

\section{CrossMark}

${ }^{1}$ Family Medicine, Hosan Hospital, Gangnam-gu, The Republic of Korea

${ }^{2}$ South Suburban Family Medicine, Denver, Colorado, USA

${ }^{3}$ Research, LD Lab, Gangnam-gu, The Republic of Korea

Correspondence to Dr June S Yang, Hosan Hospital; drisland@naver. com

\section{ABSTRACT}

Background High-sensitivity $\mathrm{C}$ reactive protein (hsCRP) is a reliable indicator of atherosclerotic diseases and is associated with hyperinsulinaemia. The purpose of this study is to examine the association between hsCRP and fasting insulin levels in Korean adults not taking medication for hypertension, dyslipidaemia or diabetes, nor provided with specific dietary advice.

Methods This cross-sectional study included 2588 subjects who participated in the 2015 Korean National Health and Nutrition Examination Survey. High-risk hsCRP was defined as hs-CRP $>2 \mathrm{mg} / \mathrm{L}$. Subjects were divided into four groups with fasting insulin $(<5,5-10$, $10-15,>15 \mathrm{ulU} / \mathrm{mL}$ )

Results The mean hs-CRP in our sample was $1.22 \mathrm{mg} / \mathrm{L}$. hs-CRP was positively associated with fasting insulin level. The occurrence of high-risk hsCRP with increasing fasting insulin levels was $7.6 \%$, $9.8 \%, 19.1 \%$ and $28.2 \%$, respectively. In comparing high-risk hs-CRP between the highest ( $>15 \mu \mathrm{lU} / \mathrm{mL}$ ) and lowest $(<5 \mu \mathrm{lU} / \mathrm{mL})$ insulin groups, the $\mathrm{OR}$ $(95 \% \mathrm{Cl})$ was 4.59 (2.77-7.58). triglycerides/highdensity lipoprotein ratio was also associated with hsCRP. Low-density lipoprotein cholesterol was not associated with hs-CRP.

Conclusions Higher level of fasting insulin is positively associated with high-risk hs-CRP.

\section{INTRODUCTION}

Several studies have shown that plasma insulin levels, fasting or after oral glucose load, are associated with increased risk of cardiovascular disease. ${ }^{1-4}$ This subject, however, has been controversial because of conflicting results through other studies. ${ }^{5-7}$ Though insulin was associated with risk factors for cardiovascular disease, it was not usually associated with cardiovascular disease itself.

An increase in high-sensitivity $\mathrm{C}$ reactive protein (hs-CRP) is a sensitive marker of systemic inflammation, and is associated with early atherosclerotic changes, thus predicting cardiovascular risk. ${ }^{8} 9$ hsCRP $>2 \mathrm{mg} / \mathrm{L}$ was defined as high risk for cardiovascular disease. ${ }^{10}$

\section{What are the new findings?}

An analysis of Korean adults not taking medicines for diabetes, dyslipidaemia and hypertension also without specific dietary advice showed that fasting insulin is positively associated with high-sensitivity $\mathrm{C}$ reactive protein (hs-CRP).

- The OR for high-risk hs-CRP in the subjects whose fasting insulin was $>15 \mu \mathrm{lU} / \mathrm{mL}$ compared with those whose fasting insulin $<5 \mu \mathrm{IU} / \mathrm{mL}$ was 4.59 .

- Triglycerides/high-density lipoprotein ratio was also associated with hs-CRP, but low-density lipoprotein cholesterol was not associated with hs-CRP.

How might it impact on clinical practice in the near future?

- Findings support an insulin-lowering lifestyle modification for preventing cardiovascular disease.

A few studies have suggested that elevated levels of plasma hs-CRP are associated with insulin resistance, hyperinsulinaemia and cardiovascular autonomic dysfunction. ${ }^{11-14}$

This study was designed to examine the association between hs-CRP and fasting insulin in Korean adults not taking medications for hypertension, dyslipidaemia or diabetes, nor provided with specific dietary advice, by analysing the sixth Korean National Health and Nutrition Examination Survey (KNHANES) performed in 2015 .

\section{METHODS \\ Patients}

We analysed the participants of the third year (2015) of KNHANES VI. KNHANES was designed to assess the health and nutritional status of adults and children over 
Table 1 Clinical characteristics

\section{Characteristics}

\begin{tabular}{|c|c|}
\hline Age & $42.95 \pm 0.37$ \\
\hline Sex (female \%) & $46.1(44.3-48.0)$ \\
\hline Body mass index $\left(\mathrm{kg} / \mathrm{m}^{2}\right)$ & $23.43 \pm 0.08$ \\
\hline Systolic BP (mm Hg) & $114.68 \pm 0.37$ \\
\hline Diastolic BP (mm Hg) & $75.09 \pm 0.25$ \\
\hline Fasting glucose(mg/dL) & $96.84 \pm 0.56$ \\
\hline Fasting insulin ( $\mu \mathrm{IU} / \mathrm{mL})$ & $7.99 \pm 0.16$ \\
\hline HbA1c (\%, DCCT) & $5.56 \pm 0.03$ \\
\hline hs-CRP (mg/L) & $1.14 \pm 0.05$ \\
\hline Total cholesterol (mg/dL) & $192.51 \pm 0.83$ \\
\hline $\mathrm{TG}(\mathrm{mg} / \mathrm{dL})$ & $140.53 \pm 3.06$ \\
\hline HDL cholesterol (mg/dL) & $51.65 \pm 0.29$ \\
\hline LDL cholesterol (mg/dL) & $115.79 \pm 0.73$ \\
\hline TG/HDL ratio & $3.98 \pm 0.17$ \\
\hline AST (IU/L) & $22.49 \pm 0.30$ \\
\hline ALT (IU/L) & $22.58 \pm 0.61$ \\
\hline Smoking, \% Never & $52.9(50.6-55.1)$ \\
\hline Ex-smoker & $20.3(18.6-22.0)$ \\
\hline Current & $25.3(22.5-28.4)$ \\
\hline Alcohol $(\%)<1 /$ month & $30.3(27.1-33.9)$ \\
\hline 1/month-1/week & $38.1(34.8-41.5)$ \\
\hline$>2 /$ week & $23.6(20.9-26.6)$ \\
\hline Exercise (\%) & $52.9(50.7-55.1)$ \\
\hline Total energy intake (kcal) & $2251 \pm 28$ \\
\hline Carbohydrate intake (\%) & $60.90 \pm 0.33$ \\
\hline Fat intake (\%) & $20.21 \pm 0.24$ \\
\hline
\end{tabular}

Data are presented with survey means \pm SE for continuous variables and weighted percentages $(95 \% \mathrm{Cl})$ for categorical variables. Exercise(\%) is defined as the percentage of people doing aerobic exercise based on whether a subject reports doing aerobic exercise more than 2.5 hours/week of moderate intensity or 1.25 hours/week of high intensity. Carbohydrate and fat intake (\%) is shown as a percentage of total energy intake.

ALT, alanine aminotransferase; AST, aspartate aminotransferase; BP, blood pressure; DCCT, Diabetes Control and Complications Trial units; HDL, high-density lipoprotein; hs-CRP, high-sensitivity $C$ reactive protein; LDL, low-density lipoprotein; TG, triglycerides.

1 year of age. Specifically, KNHANES targeted the noninstitutionalised civilian Korean population. The KNHANES VI was a cross-sectional and nationally representative survey conducted by the Division of Chronic Disease Surveillance, Korea Centers for Disease Control and Prevention from 2013 to 2015. The data collected consisted of a health interview survey, a health examination survey and a nutrition survey. A detailed description of the sampling method used was published in the 2013 KNHANES. ${ }^{15}$

In this survey, 12528 individuals in 4600 households were sampled, with 9308 of them participating in the health interviews and health examination surveys, and 8641 participating in the nutrition surveys. Among those who participated in the survey between January 2015 and December 2015, fasting insulin levels and hsCRP levels were obtained in 2656 males and 3200 females aged 10 years and older from all 16 administrative districts of South Korea. This study was restricted to participants over 20 years of age not provided with specific dietary advice, insulin injections or other medications for blood pressure, dyslipidaemia or diabetes $(\mathrm{n}=2588)$.

\section{General characteristics of the subjects}

Assessed characteristics included age, gender, anthropometric measurement and health-related behaviours. Blood pressure was manually measured by trained examiner, three times, at $30 \mathrm{~s}$ intervals, using the subject's right arm after a minimum of $5 \mathrm{~min}$ of rest in a seated position. The average of the second and third readings was considered as the final blood pressure. Height and weight were measured with the participant wearing light clothing and no shoes. Body mass index (BMI) was computed as weight $(\mathrm{kg})$ divided by the height $(\mathrm{m})$ squared. Waist circumference was measured by placing a tape measure at the midpoint between the inferior margin of the last rib and the iliac crest in the midaxillary plane, while the participant maintained a full expiratory position.

Information on health-related behaviours such as smoking status (non-smoker, ex-smoker or current smoker), alcohol intake (less than once per month, less than once per week or more than twice per week) and regular physical activity of moderate intensity (less or more than three times per week) was obtained from the health questionnaire. Moderate-intensity activities were defined as those lasting at least $10 \mathrm{~min}$ and causing a slight increase in the individual's heart rate compared with sedentary activities; table tennis, swimming, yoga and badminton were included as moderate-intensity activities, but walking was excluded.

\section{Dietary assessment}

Dietary data were collected from the KNHANES using a 24-hour recall. Intake of macronutrients was calculated based on 24-hour recall data. The ratio of energy from each macronutrient was calculated.

\section{Biochemical measurements}

Blood samples were obtained after overnight fasting. Blood samples were collected from each participant during the survey and were processed, immediately refrigerated and transported in cold storage to the Central Testing Institute in Seoul, Korea. The serum 
Table 2 Clinical characteristics of fasting insulin groups

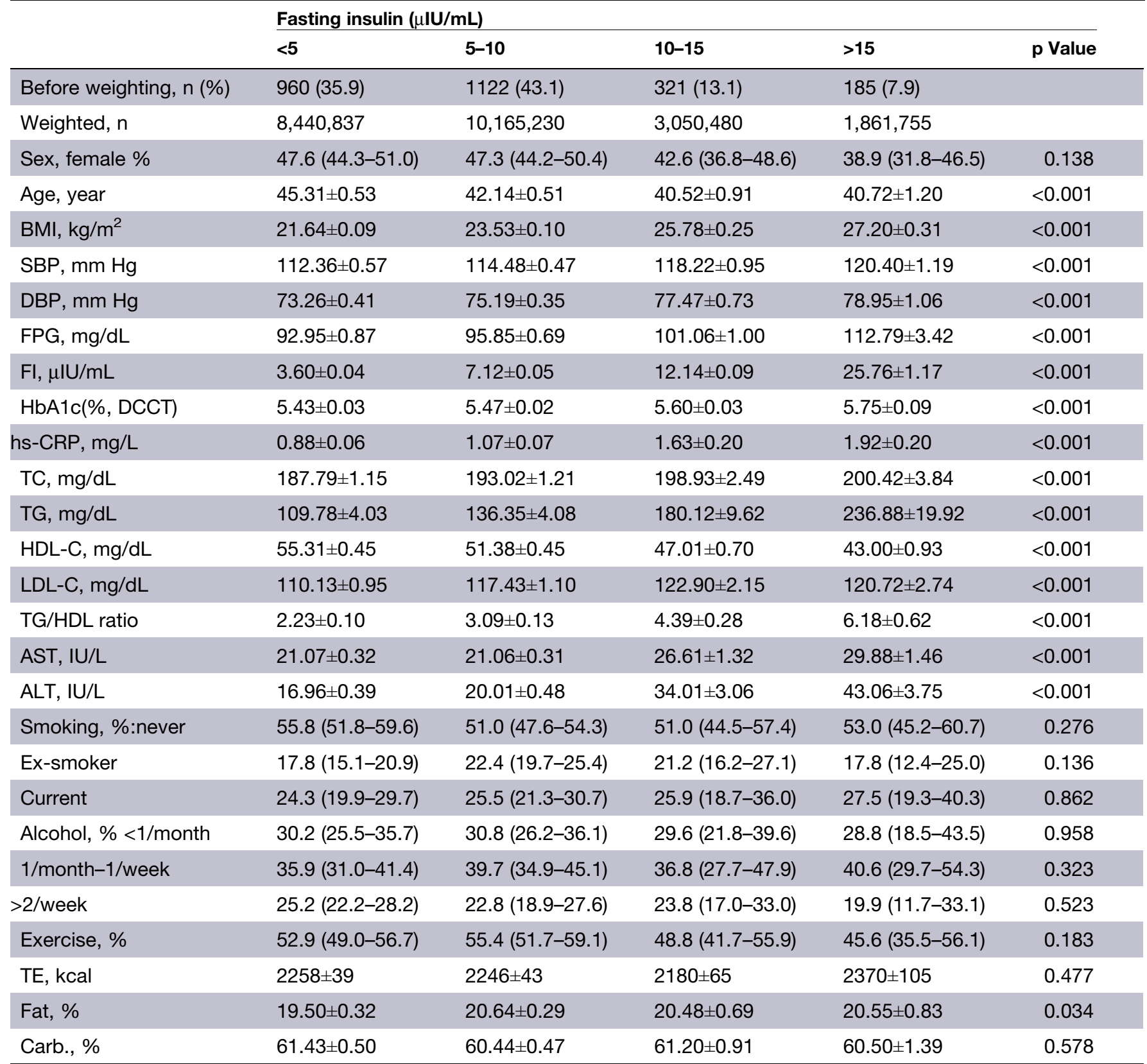

Data are presented with survey means \pm SE for continuous variables and weighted percentages $(95 \% \mathrm{Cl})$ for categorical variables. $\mathrm{p}$ Values were based on the one-way analysis of variance test and $\chi^{2}$ test.

ALT, alanine transaminase; AST, aspartate transaminase; BMI, body mass index; carb., carbohydrate; DBP, diastolic blood pressure; DCCT, Diabetes Control and Complications Trial units; FI, fasting insulin; FPG, fasting plasma glucose; HDL-C, high-density lipoprotein cholesterol; hs-CRP, high-sensitivity C reactive protein; LDL-C, low-density lipoprotein cholesterol; SBP, systolic blood pressure; TC, total cholesterol; TE, total energy intake; TG, triglycerides.

levels of total cholesterol, triglycerides (TG), highdensity lipoprotein cholesterol (HDL-C), low-density lipoprotein cholesterol (LDL-C), aspartate aminotransferase (AST) and alanine aminotransferase (ALT) were measured enzymatically using a Hitachi Automatic Analyzer 7600 (Hitachi, Tokyo, Japan). Insulin concentration was measured by immunoradiometry (INSIRMA, Bio-Source, Nivelles, Belgium).
Definition of high-risk hs-CRP and fasting insulin levels hs-CRP $>2 \mathrm{mg} / \mathrm{L}$ was defined as high-risk hsCRP. ${ }^{10}$ Subjects were divided into four groups as their insulin levels $(<5,5-10,10-15,>15 \mu \mathrm{IU} / \mathrm{mL})$.

\section{Statistical analysis}

Statistical analyses were performed using SPSS V. 23.0 (SPSS). Survey analyses were weighted to reflect 
Table 3 Mean hs-CRP, the occurrence of high-risk hs-CRP(>2 mg/L) (\%) versus fasting insulin

\begin{tabular}{|c|c|c|c|c|c|}
\hline & $<5$ & $5-10$ & $10-15$ & $>15$ & p Value \\
\hline \multicolumn{6}{|c|}{ Mean hs-CRP (mg/L) } \\
\hline Unadjusted & $0.88 \pm 0.06$ & $1.07 \pm 0.07$ & $1.63 \pm 0.20$ & $1.92 \pm 0.20$ & $<0.001$ \\
\hline Model I & $0.86 \pm 0.06$ & $1.08 \pm 0.07$ & $1.65 \pm 0.20$ & $1.94 \pm 0.20$ & $<0.001$ \\
\hline Model II & $0.86 \pm 0.06$ & $1.07 \pm 0.07$ & $1.66 \pm 0.20$ & $1.95 \pm 0.20$ & $<0.001$ \\
\hline \multicolumn{6}{|c|}{ High-risk hs-CRP (hs-CRP>2 mg/L) (\%) } \\
\hline Unadjusted & $7.92 \pm 0.97$ & $9.80 \pm 1.08$ & $19.30 \pm 2.74$ & $28.29 \pm 4.04$ & $<0.001$ \\
\hline Model I & $7.70 \pm 0.98$ & $9.88 \pm 1.08$ & $19.52 \pm 2.74$ & $28.44 \pm 4.06$ & $<0.001$ \\
\hline Model II & $7.75 \pm 0.99$ & $9.78 \pm 1.07$ & $19.68 \pm 2.73$ & $28.54 \pm 4.04$ & $<0.001$ \\
\hline
\end{tabular}

Data are presented with survey means \pm SE.

Model I: adjusted for sex, age.

Model II: adjusted for model I + alcohol, smoking, exercise, total energy intake.

hs-CRP, high-sensitivity $\mathrm{C}$ reactive protein.

complex survey sampling, unequal probabilities of selection, non-response adjustments and to produce unbiased estimates generalisable to the Korean population. Data are presented as means with $\mathrm{SE}$ for continuous variables and percentages with 95\% CI for categorical variables. An independent two-sample t test was used to compare two independent groups. We performed complex samples general linear model analysis to investigate the association between insulin and hs-CRP. The complex samples logistic regression analysis was employed to obtain the OR associated insulin and hs-CRP after adjusting for sex and age. Subjects with serum insulin $<5 \mu \mathrm{IU} / \mathrm{mL}$ were selected

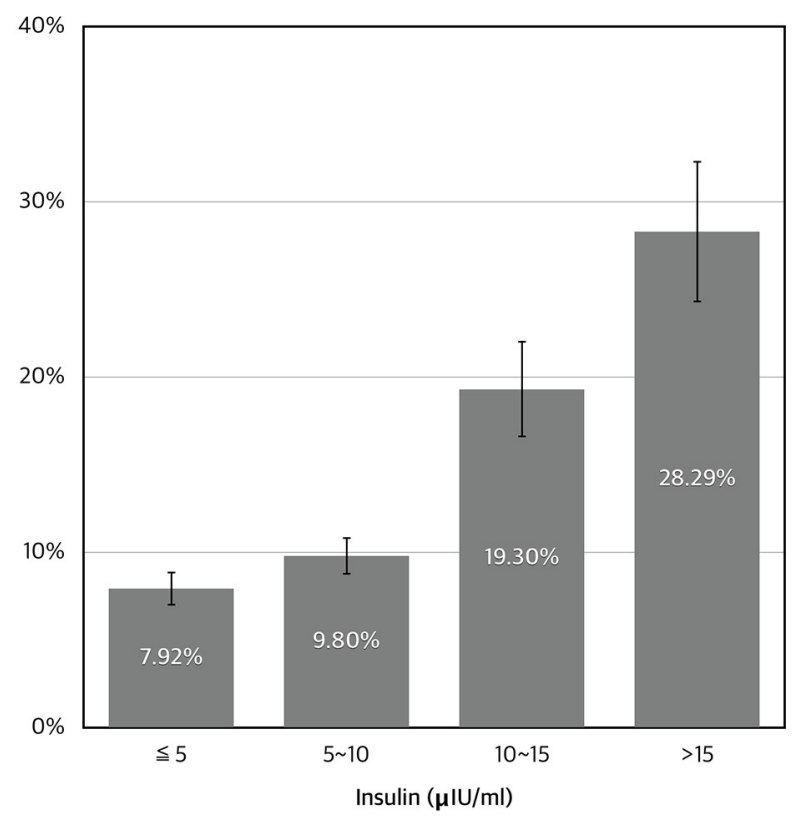

Figure 1 Occurrence of high-risk high-sensitivity $\mathrm{C}$ reactive protein ( $>2 \mathrm{mg} / \mathrm{L})$ (\%) versus fasting insulin (unadjusted). as the reference group because this was the range associated with the lowest hs-CRP. All $p$ values were two tailed and results were considered statistically significant if the $\mathrm{p}$ values were $<0.05$.

\section{RESULTS}

The cross-sectional analyses included data on 2588 participants (1162 men and 1426 women), aged $\geq 20$ years, not provided with specific dietary advice, insulin injections or other medications for high blood pressure, dyslipidaemia or diabetes. The mean age of the study participants was $42.95 \pm 0.37$. Table 1 shows the general characteristics of the study participants. LDL-C was directly measured in all of subjects.

Then, subjects were segmented into four insulin groups as follows: serum insulin $(<5,5-10,10$ $15, \geq 15 \mathrm{uIU} / \mathrm{mL}$ ). Table 2 shows subjects' characteristics according to their insulin levels. The mean or median values of body weight, BMI, waist circumference, systolic blood pressure (SBP), diastolic blood pressure (DBP), fasting glucose, HbAlc, AST, ALT, hsCRP, TG/HDL ratio, LDL-C and homeostasis model assessment of insulin resistance (HOMA-IR). Conversely, age and HDL-C decreased as fasting insulin level increased.

Table 3 shows hs-CRP relative to fasting insulin levels. hs-CRP increased as insulin level increased $(p<0.001)$. The mean of hs-CRP was $1.14 \pm 0.05$. The occurrence of high-risk hs-CRP in the lowest insulin group was $7.92 \% \pm 0.97 \%$ while those in the highest insulin group was $28.29 \% \pm 4.04 \%$ (figure 1). The OR for high-risk hs-CRP in the highest fasting insulin group compared with the lowest fasting insulin group was 4.59 (95\% CI 2.77 to 7.58) (table 4). After multiple covariates were adjusted, the OR for high-risk hs-CRP in the highest fasting insulin group was 4.78 (2.768.27). 
Table 4 The OR between fasting insulin and the occurrence of high-risk hs-CRP (>2 mg/L)

OR(95\% Cl) between fasting insulin $(\mu \mathrm{IU} / \mathrm{mL})$ and high-risk hs-CRP

\begin{tabular}{llllll}
\hline Outcomes & $<5$ & $\mathbf{5 - 1 0}$ & $\mathbf{1 0 - 1 5}$ & $>15$ & p Value \\
\hline Unadjusted & 1 (ref) & $1.26(0.89-1.79)$ & $2.78(1.79-4.33)$ & $4.59(2.77-7.58)$ & $<0.001$ \\
Model I & 1 (ref) & $1.30(0.92-1.84)$ & $2.91(1.87-4.53)$ & $4.78(2.87-7.94)$ & $<0.001$ \\
Model II & 1 (ref) & $1.27(0.89-1.81)$ & $3.02(1.90-4.80)$ & $4.78(2.76-8.27)$ & $<0.001$ \\
\hline
\end{tabular}

Participants with fasting insulin $<5 \mu \mathrm{IU} / \mathrm{mL}$ were reference group. ORs are given with $95 \% \mathrm{Cl}$ in parentheses.

Model I: adjusted for sex, age.

Model II: adjusted for model I + alcohol, smoking, exercise, total energy intake.

hs-CRP, high-sensitivity $\mathrm{C}$ reactive protein.

Table 5 The OR between TG/HDL ratio and the occurrence of high-risk hs-CRP (>2 mg/L)

OR $(95 \% \mathrm{Cl})$ between TG/HDL ratio and high-risk hs-CRP

\begin{tabular}{lllllll}
\hline Outcomes & $<2$ & $\mathbf{2 - 4}$ & $\mathbf{4 - 6}$ & $>6$ & p Value & \\
\hline $\mathrm{n}(\%)$ & $1250(49)$ & $763(29.6)$ & $280(11.4)$ & $230(10.1)$ & $<0.001$ \\
Unadjusted & 1 (ref) & $1.71(1.24-2.37)$ & $2.17(1.46-3.24)$ & $2.79(1.84-4.24)$ & $<0.001$ \\
Model I & 1 (ref) & $1.78(1.27-2.53)$ & $2.32(1.50-3.61)$ & $3.02(1.86-4.90)$ & $<0.001$ \\
\hline Model II & 1 (ref) & $1.79(1.25-2.56)$ & $2.32(1.48-3.62)$ & $2.87(1.69-4.87)$ & \\
\hline
\end{tabular}

Participants with TG/HDL ratio $<2$ were reference group. ORs are given with $95 \% \mathrm{Cl}$ in parentheses.

Model I: adjusted for sex, age.

Model II: adjusted for model I + alcohol, smoking, exercise, total energy intake.

HDL, high-density lipoprotein; hs-CRP, high-sensitivity $\mathrm{C}$ reactive protein; TG, triglycerides.

To compare the effects of fasting insulin with the association between TG/HDL ratio and hs-CRP, subjects were divided into four groups as TG/HDL ratio $<2,2-4$, $4-6$ and $>6$. The OR for high-risk hs-CRP between TG/ HDL groups were $1.71,2.17,2.79$, respectively (table 5 , $\mathrm{p}=<0.001)$.

After subjects were divided as serum levels of LDL-C $(<70,70-120,120-170,>170)$, there was no significant differences in hs-CRP among groups (table 6, $\mathrm{p}=0.748)$. LDL-C was not associated with hs-CRP. There was no significant difference between subjects lower than $70 \mathrm{mg} / \mathrm{dL}$ of LDL-C and higher than $170 \mathrm{mg} / \mathrm{dL}$.

\section{DISCUSSION}

Our results based on the Korea National Health and Nutrition Examination Survey 2015 showed that elevated fasting insulin levels were associated with increased high-risk hs-CRP $(>2 \mathrm{mg} / \mathrm{L})$. This association was independent of other lifestyle factors, including smoking, alcohol, physical activity and total energy intake.

In the Helsinki Policeman Study, age-adjusted HR for major CHD risk during 10 years comparing men in the highest area under curve insulin quintile with those in the combined four lower quintiles was 2.72 (95\% CI 1.67 to 4.42$).{ }^{3}$ In the most recent meta-analysis including 14 studies, however, the OR for CHD compared the top third of fasting insulin with the

\begin{tabular}{|c|c|c|c|c|c|}
\hline \multicolumn{5}{|c|}{ OR $(95 \% \mathrm{Cl})$ between LDL-C (mg/dL) and high-risk hs-CRP } & \multirow[b]{2}{*}{$p$ Value } \\
\hline Outcomes & $<70$ & $70-120$ & $120-170$ & $>170$ & \\
\hline$n(\%)$ & $146(5.9)$ & $1341(52.7)$ & $965(36.6)$ & $136(4.8)$ & \\
\hline Unadjusted & 1 (ref) & $0.91(0.47-1.77)$ & $0.95(0.47-1.93)$ & $1.25(0.52-3.02)$ & 0.748 \\
\hline Model I & 1 (ref) & $0.92(0.45-1.88)$ & $0.92(0.45-1.88)$ & $1.19(0.48-2.92)$ & 0.311 \\
\hline Model II & 1 (ref) & $1.10(0.55-2.21)$ & $1.12(0.53-2.37)$ & $1.35(0.53-3.44)$ & 0.917 \\
\hline
\end{tabular}

hs-CRP, high-sensitivity C reactive protein; LDL-C, low-density lipoprotein cholesterol. 
bottom third was weak, 1.12 (95\% CI 0.98 to 1.28$).{ }^{16}$ The precision of method used for measuring insulin and fasting time may be an explanation for this disparity.

On the other hand, hyperinsulinaemia was associated with multiple risk factors for CHD as obesity, hypertension, impaired glucose tolerance, type 2 diabetes, hypercholesterolaemia and hypertriglyceridaemia in the San Antonio Heart Study. ${ }^{17}$

In a cross-sectional study of 1525 Peruvian adults, CRP was positively associated with fasting insulin. ${ }^{11} \mathrm{~A}$ cross-sectional study in Japan showed that the hs-CRP level of 121 male subjects was positively associated with fasting insulin. ${ }^{12}$ A case-control study of 35 patients with type 2 diabetes showed that fasting plasma insulin was higher in the increased hs-CRP group. ${ }^{13}$ Compared with lower hs-CRP subjects, higher hs-CRP group in euglycaemic healthy young adults recorded increased insulin level during oral glucose tolerance test. ${ }^{14}$

A number of studies have reported that low-grade inflammation is a novel risk factor in all stages of atherosclerosis and acute coronary syndrome, which can be measured as hs-CRP. ${ }^{18}$ The American College of Cardiology / American Heart Association guidelines on the assessment of cardiovascular risk acknowledged hs$\mathrm{CRP}>2 \mathrm{mg} / \mathrm{L}$ as a risk assessment option. ${ }^{10}$

In our study, higher level of fasting insulin was positively associated with high-risk hs-CRP. These findings are consistent with reports from various cross-sectional, case-control and clinical trial studies of other populations. There was also a dose-response relationship among the different insulin groups. Additionally, fasting insulin was positively associated with BMI, waist circumference, SBP and DBP, fasting plasma glucose, HbAlc, total cholesterol, TG, LDL-C, TG/HDL ratio, ALT and AST. Fasting insulin was negatively associated with age and HDL-C. Many of these results are characterised as markers of metabolic syndrome. In fact, fasting insulin may be used as a marker for metabolic syndrome.

Subject group divided into different levels of LDL-C (direct measurement) were not associated with hs-CRP. This result may be in agreement with the previous observation that LDL-C was not associated with major coronary events. ${ }^{19} \mathrm{TG} / \mathrm{HDL}$ ratio is another marker for metabolic syndrome, insulin resistance and cardiovascular disease. ${ }^{20}$ Subjects divided into different TG/HDL ratio group showed an association with high-risk CRP and dose-response relationship. In case of familial hypertriglyceridaemia or familial hypercholesterolaemia, however, TG/HDL ratio cannot be a proper measure for cardiovascular risk. Fasting insulin especially may be a good option for those cases.

Our study included numerous subjects in a national survey which involved a large, nationwide, population-based sampling and also involved the collection of extensive data including potential confounders. All covariates were reliable and standardised through the use of a uniform questionnaire and surveillance protocol. Cross-sectional study design, however, is an important limitation that should be considered when interpreting the result of our study. It is difficult to determine a causal relationship between fasting insulin and hs-CRP. Whether higher level of insulin promotes minor inflammation or minor inflammation aggravates insulin resistance cannot be determined.

\section{CONCLUSION}

The results of this study demonstrate a positive association between fasting insulin level and hs-CRP in a nationally representative sample of Korean adults after controlling for confounders. Comparing high-risk hs-CRP $(>2 \mathrm{mg} / \mathrm{L})$ between the highest $(>15 \mu \mathrm{IU} / \mathrm{mL})$ and lowest $(<5 \quad \mu \mathrm{IU} / \mathrm{mL})$ insulin groups, the OR $(95 \% \mathrm{CI})$ was 4.59 (2.77-7.58). TG/ HDL ratio was also associated with hs-CRP. LDL-C was not associated with hs-CRP.

\section{Competing interests None declared}

Ethics approval This investigation was conducted according to the principles expressed in the Declaration of Helsinki. The participants of Korean National Health and Nutrition Examination Survey (KNHANES) engaged voluntarily. The KNHANES has been performed since 1998 and received ethical approval by Institutional Review Board of Korea Center for Disease Control and Prevention.

Provenance and peer review Not commissioned; externally peer reviewed.

Data sharing statement The entire data can be downloaded from "https:// knhanes.cdc.go.kr".

Open Access This is an Open Access article distributed in accordance with the Creative Commons Attribution Non Commercial (CC BY-NC 4.0) license, which permits others to distribute, remix, adapt, build upon this work noncommercially, and license their derivative works on different terms, provided the original work is properly cited and the use is non-commercial. See: http:// creativecommons.org/licenses/by-nc/4.0/

(c) Article author(s) (or their employer(s) unless otherwise stated in the text of the article) 2017. All rights reserved. No commercial use is permitted unless otherwise expressly granted.

\section{REFERENCES}

1. Pyöräla K. Relationship of glucose tolerance and plasma insulin to the incidence of coronary heart disease: results from two population studies in Finland. Diabetes Care 1979;2:131-41.

2. Welborn TA, Wearne K. Coronary heart disease incidence and cardiovascular mortality in Busselton with reference to glucose and insulin concentrations. Diabetes Care 1979;2:154-60.

3. Pyörälä M, Miettinen $\mathrm{H}$, Laakso $\mathrm{M}$, et al. Hyperinsulinemia predicts coronary heart disease risk in healthy middle-aged men: the 22-year follow-up results of the Helsinki Policemen Study. Circulation 1998;98:398-404.

4. Ducimetiere P, Eschwege E, Papoz L, et al. Relationship of plasma insulin levels to the incidence of myocardial infarction and coronary heart disease mortality in a middle-aged population. Diabetologia 1980;19:205-10.

5. Jandeleit-Dahm KA, Gray SP. Insulin and cardiovascular disease: biomarker or association? Diabetologia 2012;55:3145-3151.

6. Sarwar N, Sattar N, Gudnason V, et al. Circulating concentrations of insulin markers and coronary heart disease: a quantitative review of 19 western prospective studies. Eur Heart $J$ 2007;28:2491-7.

7. Lindberg O, Tilvis RS, Stranberg TE, et al. Elevated fasting plasma insulin in a general aged population: an innocent companion of cardiovascular diseases. J Am Geriatr Soc 1997;45:407-12. 
8. Ridker PM, Cushman M, Stampfer MJ, et al. Plasma concentration of $\mathrm{C}$-reactive protein and risk of developing peripheral vascular disease. Circulation 1998;97:425-8.

9. Ridker PM, Cushman M, Stampfer MJ, et al. Inflammation, aspirin, and the risk of cardiovascular disease in apparently healthy men. N Engl J Med 1997;336:973-9.

10. Goff DC, Lloyd-Jones DM, Bennett G, et al. 2013 ACC/AHA guideline on the assessment of cardiovascular risk: a report of the American College of Cardiology/American Heart Association Task Force on Practice Guidelines. Circulation 2014;129(25 Suppl 2):S49-73.

11. Gelaye B, Revilla L, Lopez T, et al. Association between insulin resistance and c-reactive protein among peruvian adults. Diabetol Metab Syndr 2010;2:30.

12. Hanyu O, Yoshida J, Abe E, et al. High-sensitivity CRP reflects insulin resistance in smokers. J Atheroscler Thromb 2009;16:560-7.

13. Anan F, Takahashi N, Nakagawa M, et al. High-sensitivity C-reactive protein is associated with insulin resistance and cardiovascular autonomic dysfunction in type 2 diabetic patients. Metabolism 2005;54:552-8.

14. Preethi BL, Prasanna Kumar KM, Jaisri G, et al. High-sensitivity Creactive protein a surrogate marker of insulin resistance. J Physiol Pathophysiol 2013;4:29-36.
15. Korea Centers for Disease Control and Prevention, Ministry of Health and Welfare. Korean National Health and Nutrition Examination surveys: the 6th surveys. http://knhanes.cdc.go.kr.

16. Sarwar N, Sattar N, Gudnason V, et al. Circulating concentrations of insulin markers and coronary heart disease: a quantitative review of 19 western prospective studies. Eur Heart $J$ 2007;28:2491-7.

17. Ferrannini E, Haffner SM, Mitchell BD, et al. Hyperinsulinaemia: the key feature of a cardiovascular and metabolic syndrome. Diabetologia 1991;34:416-22.

18. Libby $P$, Ridker PM, Maseri A. Inflammation and atherosclerosis. Circulation 2002;105:1135-43.

19. Sachdeva A, Cannon CP, Deedwania PC, et al. Lipid levels in patients hospitalized with coronary artery disease: an analysis of 136,905 hospitalizations in get with the guidelines. Am Heart $J$ 2009;157:111-7.

20. Kim JS, Kang HT, Shim JY, et al. The association between the triglyceride to high-density lipoprotein cholesterol ratio with insulin resistance (HOMA-IR) in the general korean population: based on the National Health and Nutrition Examination survey in 2007-2009. Diabetes Res Clin Pract 2012;97:132-8. 